\title{
Electroretinography in dogs using a fiber electrode prototype
}

\author{
A.L. Pereira ${ }^{1}$, F. Montiani-Ferreira ${ }^{2}$, V.R. Santos $^{3}$, S.R. Salomão ${ }^{3}$, C. Souza ${ }^{4}$ and A. Berezovsky ${ }^{3}$ \\ ${ }^{1}$ Clínica Veterinária Arca de Noé, Atibaia, SP, Brasil \\ ${ }^{2}$ Departamento de Medicina Veterinária, Universidade Federal do Paraná, Curitiba, PR, Brasil \\ ${ }^{3}$ Departamento de Oftalmologia, Escola Paulista de Medicina, Universidade Federal de São Paulo, São Paulo, SP, Brasil \\ ${ }^{4}$ Department of Veterinary Clinical Sciences, Washington State University, Pullman, WA, USA
}

\begin{abstract}
We compared two electroretinography (ERG) electrodes in dogs using ERG standards of the International Society for Clinical Electrophysiology of Vision (ISCEV). Ten healthy Yorkshire terrier dogs (mean age, $2.80 \pm 1.42$ years; 6 females) weighing $5.20 \pm 1.56 \mathrm{~kg}$ were evaluated using an ERG system for veterinary use. Dark- and light-adapted ERG responses were recorded using an ERG-Jet electrode and a fiber electrode prototype. The examinations were performed during 2 visits, 3 weeks apart. Both electrodes (ERG-Jet or fiber prototype) were used on each animal and the first eye to be recorded (OD $\times$ OS) was selected randomly. Three weeks later the examination was repeated on the same animal switching the type of electrode to be used that day and the first eye to be examined. The magnitude and waveform quality obtained with the two electrode types were similar for all ERG responses. ERG amplitudes and implicit times obtained from dogs using the fiber electrode prototype were comparable to those obtained with the ERG-Jet electrode for rod, maximal rod-cone summed, cone, and $30-\mathrm{Hz}$ flicker responses. The fiber electrode prototype is a low-cost device, available as an alternative instrument for clinical veterinary ERG recording for retinal function assessment.
\end{abstract}

Key words: Electroretinography; Electrode; Retina; Canine; Prototype

\section{Introduction}

The full-field electroretinogram (ERG) is a valuable electrophysiological technique routinely employed by veterinary ophthalmologists for evaluation of retinal function (1). Many clinical and surgical procedures depend on its interpretation (2). ERG is a complex recording of electric potentials originated in the retina as a response to light stimulation. The electrical waveform recorded represents the potential measured between an electrode on the surface of the cornea, usually a contact lens, and a reference electrode, that can be either in the same contact lens (bipolar lens) or on the skin anywhere around the eye, near the active electrode (monopolar lens) (3).

According to the International Society for Clinical Electrophysiology of Vision (ISCEV), for full-field standard clinical ERG, bipolar contact-lens electrodes provide the highest amplitude and most stable recordings with the active electrode. Such electrodes should be centrally transparent with an optical opening as large as possible, and incorporate a device to hold the lids apart (4). The Burian-Allen electrode meets these standards. A large speculum holds the eyelids apart and contacts the scleral surface, a smaller clear corneal contact lens is held against the cornea with a spring assembly and a circular silver wire around the circumference makes the electrical contact. The reference electrode is formed by a coating of silver granules in polymerized plastic within the surface of the scleral speculum for bipolar ERG recordings $(5,6)$.

Alternatively, electrodes such as gold-foil electrodes, skin electrodes and fiber electrodes have been used and have been shown to provide equivalent waveforms and amplitude when compared to the Burian-Allen electrode (7). Three decades ago, a less invasive monopolar fiber electrode was developed by Dawson, Trick, and Litzkow (8). The so-called DTL ${ }^{\mathrm{TM}}$ disposable electrode does not require topical anesthesia, and can be used for lengthy recording periods. The $\mathrm{DTL}^{\mathrm{TM}}$ electrode is based upon an extremely low mass conductive thread, which makes contact between the tear film of the eye and an adjacent stranded insulated electrical wire. The thread consists of a 2-cm length of spun nylon fibers impregnated with metallic silver by a proprietary method. The individual fibers are 
$12 \mu \mathrm{m}$ in diameter, and the spun thread consists of 3 to 6 fibers. A small terminal portion of wire is stripped of its insulation leaving the bare strands of copper wire to be attached to silver-coated nylon fibers (8).

Because the available electrodes have different configurations and are made of different materials, they can give slightly different responses. In addition, other intrinsic and extrinsic factors can play a role in the ERG values obtained, making it harder to define a normal range. Retinal function and dark/light adaptation are the main intrinsic factors that may affect the results of any ERG recording, followed by species, age, ocular opacity, and pupillary dilation. Some extrinsic factors include: time and intensity of light stimulation, electrode type and positioning, recording equipment and equipment settings, anesthetic protocol, and experimental conditions. All of these factors may differ among different veterinary hospitals or laboratories $(9,10)$ and it is imperative to be aware of these variables to avoid misinterpretation of the ERG results (11). Physiological factors have also been shown to alter ERG response in different species (10-12) and include: breed, individual variation, and other factors such as time of day when the ERG is recorded and body temperature. Therefore, considering all variables listed above, normal ranges should be obtained for each specific clinic or laboratory, species, type of electrode, and ERG equipment used.

Contact lens electrodes are the most widely used in veterinary ophthalmology, but cost more and can be hard to obtain. A cost-effective alternative is the use of disposable fiber electrodes. However, in many developing countries, both contact lens and disposable fiber corneal electrodes for ERG recording are only available through importation, which can be very time-consuming and expensive.

Developing and validating an alternative, cost-effective fiber electrode to be tested in dogs can have a tremendous impact on veterinary medicine and veterinary electrophysiology in the developing countries where electrodes are not easily obtained. Recently, we have developed and tested a prototype of a disposable fiber electrode to be used in both human and veterinary medicine. This instrument was validated in healthy human subjects and human patients with retinal dystrophies (3).

The purpose of the present study was to compare fullfield ERG responses recorded in dogs with the monopolar ERG-Jet electrode to those obtained with a fiber electrode prototype, following the ERG standards of ISCEV.

\section{Material and Methods}

\section{Animals}

Ten healthy Yorkshire terrier dogs (mean age, $2.80 \pm$ 1.42 years; 4 males and 6 females) weighing 2.50 to $7.30 \mathrm{~kg}$ (mean, $5.20 \pm 1.56 \mathrm{~kg}$ ) were evaluated using an ERG system specially designed for veterinary use (BPM
200, Retinographics, USA). Inclusion criteria were good general health and normal findings on ophthalmic examination that included: menace response, dazzle reflex, pupillary light reflex, Schirmer tear test, tonometry, and fundoscopy. All animal procedures were conducted according to the ARVO Statement for the Use of Animals and the guidelines of the Research Ethics Committee at Universidade Federal de São Paulo, Brazil. All experimental subjects were privately owned pets, and informed consent was obtained from their owners. All animals were fasted for at least $8 \mathrm{~h}$ (water and food) before performing the ERG recordings.

\section{Room conditions}

Tests were carried out in a surgical suite and no light from an external source could enter the room. A dim red light was the only light source present in the room. The animals were placed on top of a surgical table that was covered with a rubber mat to minimize electrical interference.

\section{Anesthetic protocol and dark adaptation}

Maximal pupillary dilation was obtained by applying 1 drop of $1 \%$ tropicamide (Mydriacyl ${ }^{\circledR}$, Alcon, Brazil) and $10 \%$ phenylephrine (Allergan, Brazil) to the test eye every 10 min (3 times) prior to the beginning of the ERG session. As soon as pupillary dilation was achieved the eyes were covered with bandaging material and a black band. After covering the eyes, an intramuscular injection of $0.044 \mathrm{mg} / \mathrm{kg}$ atropine (Ariston, Brazil) was administered. At the end of $15 \mathrm{~min}$, following the atropine administration, an intravenous injection of a tiletamine/ zolazepam combination (Zoletil ${ }^{\circledR}$, Virbac, Brazil) at a dose of $5 \mathrm{mg} / \mathrm{kg}$ was administered and chemical restraint was achieved after $10 \mathrm{~min}$. The animal was positioned on the table and as soon as the 30 min for dark adaptation were completed the eyes were uncovered. At that time, if the ERG-Jet electrode was used, two drops of $0.5 \%$ proparacaine hydrochloride (Alcaine ${ }^{\circledR}$, Alcon) were used as a local anesthetic for the corneal surface and the electrode was positioned. A drop of $2 \%$ methylcellulose (Ophthalmos, Brazil) was also applied to the inner surface of the electrode to protect the cornea and to ensure good electrical contact. When the prototype was used, the use of a local anesthetic and methylcellulose was not necessary and only saline was applied to the eye throughout the exam to maintain the cornea lubricated.

\section{ERG equipment/electrodes}

Standard ERG rod and cone responses were recorded from fully dilated pupils using the monopolar ERG-Jet electrode (Fabrinal SA, Switzerland) and the microfiber electrode prototype (patent pending, Brazilian Institute of Industrial Property \#PI0602186-7).

The prototype used here is a modified fiber electrode based on the same principles of the DTL ${ }^{\mathrm{TM}}$ electrode. In this prototype, the fibers are made from a textile material 
(X-Static ${ }^{\circledR}$ Silver Antimicrobial Technology, Noble Biomaterials, Inc., USA) covered with 99.8\% metallic silver, which impairs oxidation and which has been clinically proven to inhibit the growth of bacteria directly on its surface. The individual fibers are $7 \mu \mathrm{m}$ in diameter, and the spun thread consists of 10 fibers. This electrode seems to be more flexible, sterile and can provide more comfort to the subject due to its thinner threads and easy fit to the conjunctival sac. The ends have small sponges $(1.0 \times 1.0 \mathrm{~cm})$ covered with epoxy cement, which is nonconductive and can function as an insulator. The sponges are used to secure the electrode to the medial and lateral canthi by means of a double-sided adhesive tape (3).

The ERGs were obtained using a standard digital electrographic BPM-200 instrument (Retinographics Inc., USA), a portable system for veterinary use. This device consists of software that can be run on a PC and hardware including: amplifiers and filters with a fixed 6-dB/ octave high-pass filter at $0.3 \mathrm{~Hz}$ and a 48-dB/octave Bessel low-pass filter at $560 \mathrm{~Hz}$ and a white light-emitting diode (LED) array light stimulator. The computer software allows storage and averaging of individual waveforms.

\section{Electrode selection and positioning}

The type of electrode (ERG-Jet or fiber prototype) to be used on each animal on day 1 was selected randomly. The first eye to be recorded $(O D \times O S)$ was also selected randomly. Three weeks later the exam was repeated on the same animal changing the type of electrode to be used and the first eye to be examined. Both eyes were examined on each day. The reference electrode was positioned on the skin $1 \mathrm{~cm}$ near the lateral canthus and the ground electrode near the occipital region, as shown in Figure 1.

\section{ERG exam}

When the ERG-Jet was used, the electrode itself was responsible for keeping the eyelids opened and when the prototype was used the eyelids were kept open manually. Whenever artifacts were present, the computer software automatically rejected the waves. The reading would not continue unless the position of the electrode was adjusted

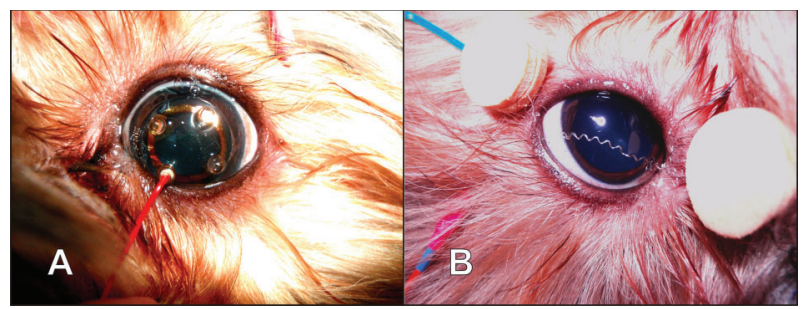

Figure 1. The electroretinogram (ERG) electrodes used in this study were positioned in the eye prior to the ERG procedure. $A$, ERG-Jet electrode (monopolar electrode). B, Fiber electrode prototype (monopolar electrode). and an appropriate wave could be recorded by the equipment. After the electrode was positioned in the eye to be examined first and the reference electrode was also in place, the diffuse and uniform light stimulator was positioned $7.5 \mathrm{~cm}$ from the cornea directed along the visual axis, according to manufacturer instructions. The spectrum of the white LED was centered at $500 \mathrm{~nm}$. Stimulus calibration was provided by the manufacturer according to ISCEV standards. Specifically, the stimulus level for rod function was 0.01 candela.s. $\left(\mathrm{m}^{-2}\right)^{-1}$ and for maximal intensity light was 2.5 candela $\cdot \mathrm{s}^{-1} \cdot\left(\mathrm{m}^{-2}\right)^{-1}$ in the dark-adapted eye. Cone function was recorded in two ways: single-flash cone response using standard flash with an intensity of 2.5 candela $\cdot \mathrm{s} \cdot\left(\mathrm{m}^{-2}\right)^{-1}$ after light adaptation from an external light [30 candela $\cdot s \cdot\left(\mathrm{m}^{-2}\right)^{-1}$ ] and flicker cone at $30-\mathrm{Hz}$ according to the ISCEV protocol for humans. ERG responses were recorded in the following order: a) dark-adapted rod response, with an inter-stimulus interval of $2 \mathrm{~s}$; b) mixed dark-adapted rod and cone response with an inter-stimulus interval of $10 \mathrm{~s}$; c) after $10 \mathrm{~min}$ of light adaptation the cone response was recorded with an inter-stimulus interval of $2 \mathrm{~s}$, and $\mathrm{d}$ ) photopic flicker $(30 \mathrm{~Hz})$ cone responses were recorded. Sixteen responses were computer averaged for each step of this protocol, except for the $30-\mathrm{Hz}$ flicker for which 50 responses were averaged.

After completion of dark- and light-adapted stimuli, each response was analyzed for a- and b-wave amplitudes and implicit times. The amplitude of the a-wave was calculated from the baseline to the first negative deflection, and the amplitude of the b-wave was measured from the trough of the a-wave to the positive peak of the bwave. Implicit times of a- and b-waves were calculated from the onset of the light stimulus to the peak of the aand b-waves, respectively (1). The amplitude of flicker ERG was measured from the trough to the peak. The implicit time was measured from each stimulus onset to the corresponding peak (2). Statistical analyses using the paired $t$-test were performed to compare parameters of amplitude $(\mu \mathrm{V})$ and implicit time (ms) for the ERG responses.

\section{Results}

ERG recordings from the right eye of all Yorkshire terrier dogs included in the study were analyzed to compare the performance of the two electrodes. Figure 2 shows representative waveforms obtained with the ERG-Jet electrode (left panels) and the fiber electrode prototype (right panels) from a single subject. The magnitude and waveform quality obtained with the two electrodes were similar for all ERG responses. Table 1 reports the mean values \pm SD for amplitude and implicit times for both the ERG-Jet electrode and the fiber electrode prototype for rod, maximal response, light-adapted cone, and $30-\mathrm{Hz}$ flicker responses. Box plots for the distribution of amplitudes and 

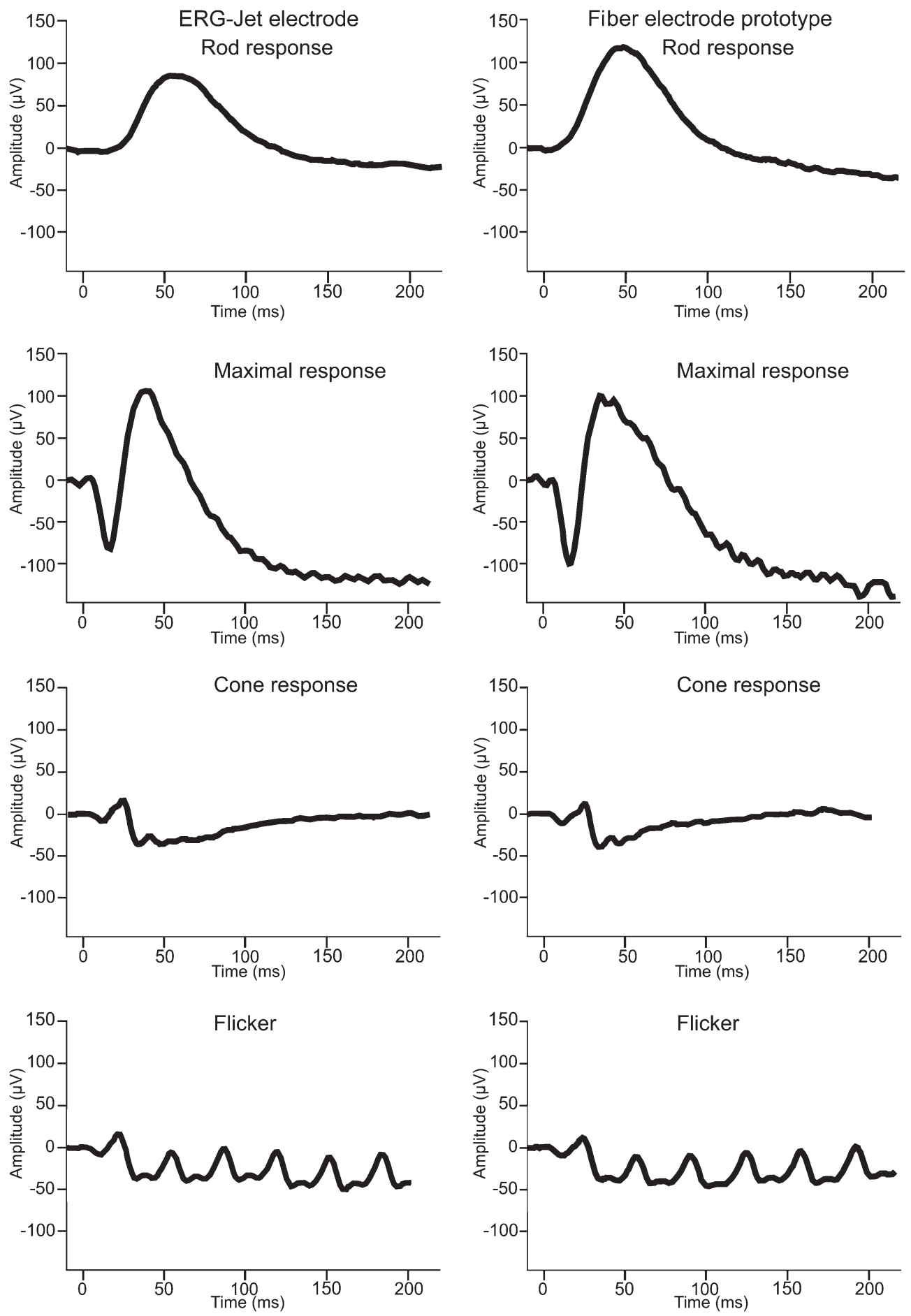

Figure 2. Representative electroretinogram (ERG) responses of a healthy Yorkshire terrier dog. Left panels, ERG recordings obtained with the ERG-Jet lens electrode. Right panels, ERG recorded with the fiber electrode prototype.

implicit times of the healthy Yorkshire terrier group studied using the ERG-Jet lens electrode and the fiber electrode prototype are shown in Figure 3.
Responses recorded with the fiber electrode prototype were comparable to those obtained with the ERG-Jet electrode. ERG a- and b-wave amplitudes and implicit 
Table 1. Comparison of the amplitudes and implicit time obtained following a standardized electroretinogram (ERG) with the ERG-Jet lens electrode and with the fiber electrode prototype.

\begin{tabular}{ccc}
\hline & $\begin{array}{c}\text { ERG-Jet electrode } \\
(\mathrm{n}=10)\end{array}$ & $\begin{array}{c}\text { Fiber electrode } \\
(\mathrm{n}=10)\end{array}$ \\
\hline $\begin{array}{c}\text { Amplitude }(\mu \mathrm{V}) \\
\text { Rod response } \\
\text { a-wave }\end{array}$ & $7.15 \pm 7.04$ & $9.79 \pm 6.40$ \\
b-wave & $94.75 \pm 34.97$ & $101.35 \pm 35.6$ \\
Maximal & & \\
a-wave & $92.68 \pm 42.11$ & $68.79 \pm 24.03$ \\
b-wave & $197.68 \pm 49.85$ & $159.42 \pm 43.30$ \\
Cone response & & \\
a-wave & $10.28 \pm 4.28$ & $11.18 \pm 13.91$ \\
b-wave & $36.65 \pm 11.74$ & $35.78 \pm 14.81$ \\
30-Hz flicker & & \\
b-wave & $37.65 \pm 15.02$ & $41.01 \pm 15.40$ \\
Implicit time $(\mathrm{ms})$ & & \\
Rod response & & \\
a-wave & $16.32 \pm 4.75$ & $16.95 \pm 2.76$ \\
b-wave & $53.87 \pm 5.85$ & $57.37 \pm 4.85$ \\
Maximal & & \\
a-wave & $11.75 \pm 1.02$ & $11.88 \pm 1.44$ \\
b-wave & $28.92 \pm 4.26$ & $30.07 \pm 3.02$ \\
Cone response & & \\
a-wave & $11.50 \pm 0.79$ & $12.43 \pm 2.27$ \\
b-wave & $26.25 \pm 2$ & $27.95 \pm 3.95$ \\
30-Hz flicker & & \\
b-wave & $26.3 \pm 2$ & $26.52 \pm 1.67$ \\
\hline
\end{tabular}

Data are reported as means \pm SD. There were no statistically significant differences (paired $t$-test)

times obtained from healthy Yorkshire terrier dogs using the fiber electrode prototype were not significantly different from those obtained with the ERG-Jet lens electrode for rod response, maximal response, cone response, and $30-\mathrm{Hz}$ flicker using paired $t$-tests. A trend without statistical significance for slower b-wave implicit time for rod, maximal, cone, and $30-\mathrm{Hz}$ flicker responses was observed with the prototype electrode compared to the ERG-Jet electrode.

\section{Discussion}

The purpose of the present study was to compare the fiber electrode prototype and ERG-Jet electrodes in the same eye of the same animal. The results showed that the ERG waveforms obtained by the two electrodes were remarkably similar for rod, maximal response, lightadapted cone, and $30-\mathrm{Hz}$ flicker responses. No statistically significant differences were found in ERGs obtained by the two electrodes for any of the parameters studied. Although electrode positioning is an important factor when evaluating ERG responses, it is not the only one that
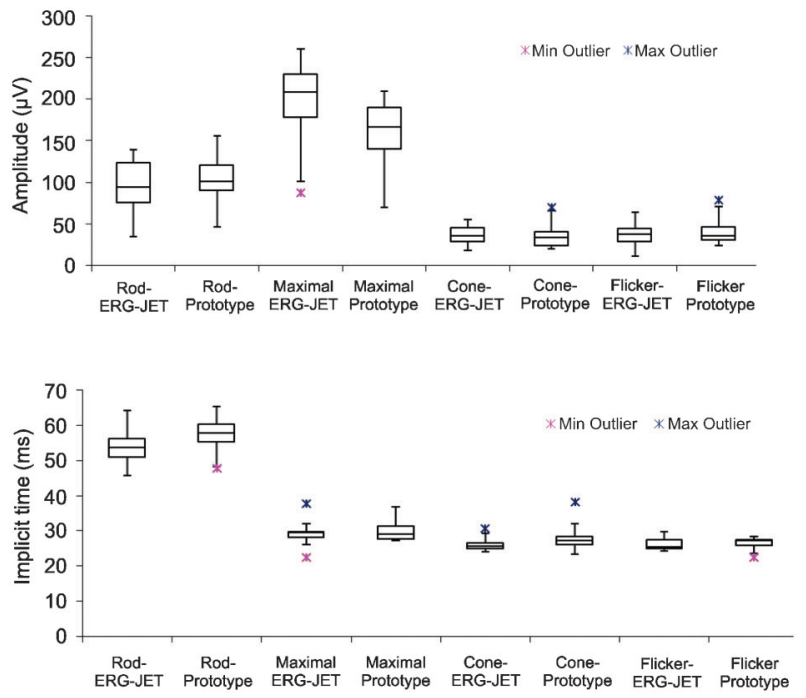

Figure 3. Box plot diagrams showing amplitude $(\mu \mathrm{V})$ in the upper panel and b-wave implicit time ( $\mathrm{ms}$ ) in the lower panel of a healthy Yorkshire terrier dog group for each step of the ISCEV standard protocol using the ERG-Jet lens electrode and the fiber electrode prototype. ERG = electroretinogram; ISCEV = International Society for Clinical Electrophysiology of Vision.

needs to be taken into consideration. It has been shown that electrode conductance characteristics can also play a role in the responses obtained $(11,12)$. In a study conducted on humans, it was found that under scotopic conditions the ERG-Jet electrode recorded a significantly larger b-wave in response to brighter flashes when compared to the DTL ${ }^{\mathrm{TM}}$ fiber electrode and therefore the two electrodes could not be used interchangeably (13). Because of the discrepancies shown by previous studies when comparing the responses recorded by different electrode types, our study focused on comparing the ERG-Jet electrode and a prototype fiber electrode. In a recent study conducted on humans, our prototype was compared to the $\mathrm{DTL}^{\mathrm{TM}}$ fiber electrode and very similar responses were obtained for all parameters (3).

In the present study, the ERG stimuli were not performed using a Ganzfeld dome, since a portable system designed for veterinary use at a more affordable price was utilized. This same system was utilized in previous veterinary studies with good success (11). The Ganzfeld dome is recommended by ISCEV due to the uniformity of the stimulus provided by this equipment, but alternative light stimulators can be used as long as they are calibrated and the stimulation meets the requirements established by the ISCEV and adequate ERG recordings are obtained. Although the Ganzfeld dome was not utilized in the present study, adequate ERG recordings were obtained with the portable system used.

In the present study, we used the anesthetic agent tiletamine-zolazepam, a combination of a dissociative anesthetic and a minor tranquilizer. This combination was 
chosen because it achieves sufficient immobilization and muscle relaxation, there is no globe rotation and the eyes remain open, all desirable effects when performing an ERG (13). All dogs used in the present study maintained a central eye position and no adverse effects or complications related to the anesthetic protocol were observed.

Many kinds of electrodes have been used for human and veterinary patients. In humans, when compared with a contact lens electrode, the fiber electrode is better tolerated by children because it is less traumatizing. This fiber prototype electrode may decrease the risk of corneal abrasion and seems to be a good alternative to corneal electrodes. Corneal electrodes require corneal anesthesia and may result in occasional corneal or conjunctival abrasions in agitated patients with exaggerated eye movements. A previous study comparing the DTL ${ }^{\mathrm{TM}}$ fiber electrode and the ERG-Jet electrode reported that the ERG-Jet electrode tended to fall out of the eye and had to be re-inserted several times, increasing the chances of corneal or conjunctival abrasions and prolonging the

\section{References}

1. Jeong MB, Son WG, Park YW, Kim SE, Park SA, Seo KM. Comparison of two electroretinography systems used in dogs: the HMsERG and the RETIport. J Vet Med Sci 2011; 73: 431-438, doi: 10.1292/jvms.10-0197.

2. Marmor MF, Fulton AB, Holder GE, Miyake $Y$, Brigell M, Bach M. ISCEV Standard for full-field clinical electroretinography (2008 update). Doc Ophthalmol 2009; 118: 69-77, doi: 10.1007/s10633-008-9155-4

3. Berezovsky A, Pereira JM, Salomão SR, Santos VR, Schor $P$. Validation of a new fiber electrode prototype for clinical electroretinography. Arq Bras Oftalmol 2008; 71: 316-320, doi: 10.1590/S0004-27492008000300003.

4. Hennessy MP, Vaegan. Amplitude scaling relationships of Burian-Allen, gold foil and Dawson, Trick and Litzkow electrodes. Doc Ophthalmol 1995; 89: 235-248, doi: 10.1007/BF01203377.

5. Burian HM, Allen L. A speculum contact lens electrode for electroretinography. Electroencephalogr Clin Neurophysiol 1954; 6: 509-511, doi: 10.1016/0013-4694(54)90070-X.

6. Gjotterberg M. Electrodes for electroretinography. A comparison of four different types. Arch Ophthalmol 1986; 104 : 569-570, doi: 10.1001/archopht.1986.01050160125027.

7. Kuze M, Uji Y. Comparison between Dawson, Trick, and Litzkow electrode and contact lens electrodes used in clinical electroretinography. Jpn J Ophthalmol 2000; 44: duration of the test compared to the $\mathrm{DTL}^{\mathrm{TM}}$ fiber electrode (12). We did not experience this problem in our study.

The ERG waveforms obtained in the present study demonstrate that the fiber electrode prototype can be utilized to evaluate retinal function in veterinary practice, in dogs. Our findings clearly indicate that the fiber electrode prototype is adequate for ERG recording in healthy dogs. It is a low-cost device with an estimated manufacturing cost reduced by approximately $1 / 3$ when compared with the imported fiber electrode (DTL $\left.{ }^{T M}\right)$. Further studies using this new fiber electrode prototype in other canine breeds and different species are desirable to extend its clinical usefulness in veterinary medicine.

\section{Acknowledgments}

Research supported by FAPESP (\#04/02669-6 and \#05/56459-5) and CNPq (\#474251/2009-8). A. Berezovsky and S.R. Salomão are recipients of CNPq research fellowships.
374-380, doi: 10.1016/S0021-5155(00)00177-5.

8. Dawson WW, Trick GL, Litzkow CA. Improved electrode for electroretinography. Invest Ophthalmol Vis Sci 1979; 18 988-991.

9. Ekesten B. Electrodiagnostic evaluation of vision. In: Gelatt KN (Editor), Veterinary ophthalmology. 4th edn. Oxford: Blackwell Publishing Ltd.; 2007. p 520-535.

10. Lee JS, Kim KH, Jang HY, Lee B, Kim JY, Jeong SW. The normal electroretinogram in adult healthy Shih Tzu dogs using the HMsERG. J Vet Sci 2009; 10: 233-238, doi 10.4142/jvs.2009.10.3.233

11. Mentzer AE, Eifler DM, Montiani-Ferreira F, Tuntivanich $\mathrm{N}$, Forcier JQ, Petersen-Jones SM. Influence of recording electrode type and reference electrode position on the canine electroretinogram. Doc Ophthalmol 2005; 111: 95106, doi: 10.1007/s10633-005-4517-7.

12. Yin $H$, Pardue MT. Performance of the DTL electrode compared to the jet contact lens electrode in clinical testing. Doc Ophthalmol 2004; 108: 77-86, doi: 10.1023 B:DOOP.0000018395.78512.4e

13. Norman JC, Narfstrom K, Barrett PM. The effects of medetomidine hydrochloride on the electroretinogram of normal dogs. Vet Ophthalmol 2008; 11: 299-305, doi: 10.1111/j.1463-5224.2008.00650.x. 